Strengths and Weaknesses of Meta-Analyses

Katherine S. Corker

Grand Valley State University

Chapter draft (January 1, 2020); Comments and feedback welcome at k.corker@gmail.com.

In press: Research Integrity in the Behavioral Sciences

L. Jussim, S. Stevens, \& J. Krosnick (Eds.)

Word count: 9,867 (excluding cover page and 90 references: 7,601) 


\section{Strengths and Weaknesses of Meta-Analyses}

How can researchers and policymakers best come to conclusions about the state of evidence in a research literature? The traditional narrative literature review has largely been supplanted in the social sciences by a quantitative technique known as meta-analysis. Metaanalysis summarizes evidence in a research area by taking an average of effect sizes. Effect sizes are weighted prior to averaging, usually using some indicator of precision of an effect size estimate. Researchers can also assess the degree of variability in effects across studies and assess possible explanations for that variability.

Armed with support from meta-analyses, social scientists can refine theoretical models, suggest future research studies, and craft evidence-based policies (Chan \& Arvey, 2012). Furthermore, meta-analysis promises a certain degree of objectivity that traditional reviews do not. If a literature search is comprehensive, the meta-analyst is simply left to compute the relevant statistics and average them. Simple, right?

Yet, the quality of the summary that results from a meta-analysis depends crucially on the quality of the included studies. In light of recent concerns about questionable research practices (John et al., 2011), publication bias (Malhotra \& Simonovits, 2014), and problems replicating research (Open Science Collaboration, 2015), scholars may wonder how informative metaanalyses actually are and whether they are worth the effort to conduct.

The current chapter briefly reviews the history of meta-analysis before turning to an assessment of its strengths and weaknesses, as well as considerations of alternatives to and modifications of traditional meta-analysis. It closes with recommendations to make metaanalyses more informative.

\section{History of Meta-Analysis in the Social and Behavioral Sciences}


It may come as a surprise that modern meta-analysis techniques were just developed in the mid-1970s - only a little over 40 years ago. Although earlier techniques had been derived to combine $p$-values (Fisher, 1932, as cited in Chalmers, Hedges, \& Cooper, 2002), correlation coefficients (Pearson, 1904), and values from contingency tables (Mantel \& Haenszel, 1959) from multiple studies, these techniques were not often used. Modern techniques involving synthesis of standardized effect sizes were developed only recently (Borenstein, Hedges, Higgins, \& Rothstein, 2009).

At its core, meta-analysis is not a complicated technique: it is simply an average of study effect sizes, weighted by a measure of study precision. Using the principal of aggregation, the meta-analyst assumes that idiosyncrasies and unreliability associated with each small individual study are averaged out, and a more precise - presumably more accurate - estimate is rendered in the aggregate. Beyond an average effect size, meta-analysis also offers the chance to measure variability (termed heterogeneity in meta-analysis lingo) in effect sizes and test for moderators that explain this variability (Borenstein et al., 2009).

Although Gene Glass was the first to coin the term "meta-analysis" to refer to the quantitative synthesis of effect sizes, several scholars independently derived meta-analytic techniques around the same time (Glass, 2015; Schmidt, 2015; Rosenthal, 2015). Glass and his contemporaries derived meta-analysis out of necessity: ever increasing numbers of original studies in the post World War II era meant that bodies of literature could no longer be adequately summarized using traditional narrative reviews. Subsequently, meta-analysis spread from psychology and education and permeated the biomedical and social sciences.

\section{Promise and Strengths of Meta-Analytic Approaches}

Early on, meta-analysis promised that it could serve as an objective arbiter of evidence, 
helping a researcher or policy maker to see the big picture in a body of literature. Individually fallible studies could be combined to give a summary conclusion that was closer to the Truth (Hunt, 1990).

The objective nature of the technique was important. A key promise was that a comprehensive, quantitative synthesis would be less sensitive to cherry picking and other biases than traditional narrative reviews (but see Ferguson, 2014). Furthermore, because techniques emphasized effect size estimation (with a focus on magnitude of effects), meta-analysis promised to reduce the emphasis on the problematic yes-no thinking that reliance on null hypothesis significance testing seemingly encouraged (Rosenthal, 2015).

Belief in the ability of meta-analysis to fulfill this early promise has waxed and waned in the 40 years since its modern debut. Almost immediately, challengers and skeptics arose who criticized meta-analysis and its premise (e.g., Eysenck, 1978), but in recent years, meta-analysis has enjoyed an enviable position at the peak of a hierarchy of evidence (Borgerson, 2009), representing the highest possible quality of evidence (perhaps the "platinum" standard of evidence, but see Stegenga, 2011).

In the last few years, however, some of the old doubts have begun to surface again. There is a growing realization that the early recognized problem of publication bias threatens to completely undermine meta-analytic synthesis. Additionally, new challenges have arisen with the recognition that researchers may not be fully reporting the extent of exploration that has created a published finding (Kerr, 1998; Nelson, Simmons, \& Simonsohn, 2011). I explain these challenges to the validity of meta-analysis in detail before examining alternative methods of evidence synthesis, as well as recommendations for valid, informative, and reproducible use of meta-analysis. 


\section{Pitfalls of Meta-Analysis in the Context of Reproducibility Issues and Questionable}

\section{Research Practices}

Publication bias. A fundamental assumption behind meta-analysis is that the selection of studies for inclusion in the analysis is comprehensive or, at the least, unbiased. That is, each study is imagined to be a member of a broader population of studies, and in order for the sample average of that population to be an unbiased estimate, the selection rule for inclusion must itself be unbiased. In principle, this means that meta-analysts must attempt to identify and include all studies that have been conducted on a topic (or at least to sample randomly within all studies).

A major threat to the unbiased inclusion of studies in meta-analyses is publication bias (Rosenthal, 1979; Sterling, 1959). Because the publication process serves as a filter through which some studies are generally admitted into the scientific record and other studies are left to languish in "file drawers," studies in the published literature are an unrepresentative sample of studies that have been conducted on a topic. There are many reasons for the non-publication of studies, but lack of statistical significance is one of the major contributors (Scherer, Langenberg, \& von Elm, 2007; Fanelli, 2010; O’Boyle, Banks, \& Gonzalez-Mulé, 2017).

Selection on statistical significance creates a situation in which published effect sizes are overestimates of true population effect sizes. ${ }^{1}$ Overestimation increases as the size of studies in the meta-analysis shrinks and as the true effect size under investigation goes to zero (Bakker et al., 2012; van Assen et al., 2015). Effects on heterogeneity are more complex (Augusteijn et al. 2019). When combined with low statistical power, there may even be a situation in which statistically significant published results are more likely to be false positives than true positives

\footnotetext{
${ }^{1}$ Publication bias can also affect heterogeneity estimates in somewhat unpredictable ways (see Augusteijn et al., 2019). These authors summarize results stating: "the Q-test of homogeneity and heterogeneity measures $H^{2}$ and $I^{2}$ are generally not valid when publication bias is present" (p. 1).
} 
(Ioannidis, 2005), which implies that meta-analytic summaries may not just be overestimates, but instead they may sometimes indicate that an effect "exists" when it actually does not.

A poignant example of this situation comes from a highly influential phenomenon from social psychology known as ego depletion. The idea behind ego depletion is that willpower or self-control is a limited resource and drawing on this resource depletes it. Thus, initial acts of self-control should predict weaker self-control on later tasks. An initial meta-analysis (Hagger et al., 2010) amassed 198 studies on this phenomenon (total sample size $=10,782$ ) and showed a medium to large average effect size with narrow confidence interval (Cohen's $d=0.62,95 \% \mathrm{CI}$ : $[0.57,0.67])$. Almost no matter how the independent variable was manipulated or the dependent variable was measured, a medium to large effect size was observed, attesting to its generalizability. But a 2014 re-analysis (Carter \& McCullough, 2014) of the meta-analytic data with various bias correction techniques suggested the true effect size could be zero, and this possibility was later shown to be consistent with results from a large scale pre-registered replication attempt (Hagger et al., 2016), which found null results (Cohen's $d=0.04,95 \%$ CI: $[-0.07,0.15])^{2}$

How could the initial meta-analysis have drawn such different conclusions from the preregistered replication? A strong possibility is that the published literature was biased because it was an incomplete record of the studies that had been done - null or negative results went unpublished. It is notable that the initial meta-analysis (Hagger et al., 2010) did not attempt to locate unpublished studies for inclusion in the analysis, but perhaps even if it had, it is unlikely that all studies would have been located and that their partial inclusion would have been enough to fully eliminate the pernicious effects of publication bias.

\footnotetext{
${ }^{2}$ n.b., Work continues on the topic of ego depletion, both methodological and theory related (e.g., Friese, Loschelder, Gieseler, Frankenbach, \& Inzlicht, 2019; Vohs, 2018).
} 
The issue of study selection was an early point of contention between meta-analysts and critics. Smith and Glass (1977) amassed over 400 studies investigating the effectiveness of psychotherapy, taking great care to conduct a comprehensive search of existing studies. Eysenck (1978) saw this comprehensiveness as a weakness and accused Smith and Glass of ignoring study quality in study selection: "A mass of reports — good, bad, and indifferent—are fed into the computer in the hope that people will cease caring about the quality of the material on which the conclusions are based" (p. 517). Glass and Smith (1978) retorted that "connoisseurs' distinctions about which studies are 'best' and which ought to be discarded would lead, in this instance, to a profligate dismissal of hundreds of findings" (p. 518).

Issues related to the selection of studies for inclusion in meta-analyses have not abated in the years since these early debates. Some sources advise meta-analysts to be as inclusive as possible in their literature searches, scouring conference abstracts, dissertation databases, and personally contacting authors likely to have file-drawered studies (Sterne, Egger, Moher, \& Boutron, 2017). Others recommend evaluating study quality (see Sanderson, Tatt, \& Higgins, 2007 for a variety of such systems) to exclude studies that do not meet certain quality or design standards (e.g., L'Abbé, Detsky, \& O'Rourke, 1987), to include quality as a meta-analytic moderator (Borenstein et al., 2009), or to weight studies by quality (Doi \& Thalib, 2008). Sensitivity analyses (in which a variety of different corrections are performed to allow metaanalysts to determine whether results are sensitive to correction method) have also been recommended (Applebaum, Cooper, Kline, Mayo-Wilson, Nezu, \& Rao, 2018). In the absence of mandatory prospective study registration, it seems unlikely that any literature search will ever be truly comprehensive, but as I discuss below (see Systematic Review), there are best practices that meta-analysts can follow to attempt to mitigate selection bias. 
Questionable research practices. Researchers do not only select which effects to include or exclude from a publication. They may also tweak, edit, and craft the specific analyses that are ultimately included in their published papers in the service of obtaining statistical significance. Many (often ambiguous) study design decisions must also be made along the way (e.g., how to best measure a variable, which outliers to exclude). Because it is not always clear cut which design or analysis variant is correct or best, researchers have a large amount of leeway to make choices that favor obtaining statistical significance, but ultimately inflate the number of false positive results. These behaviors have been variously termed questionable research practices (QRPs), p-hacking, exploiting researcher degrees of freedom, and the garden of forking paths (see Simmons, Nelson, \& Simonsohn, 2011; Gelman \& Loken, 2013).

For the meta-analyst, the presence of QRPs in the research literature means that metaanalytic data are likely to be biased. Exactly how much effect sizes are biased under various conditions is an active and ongoing area of research (Simonsohn, Nelson, \& Simmons, 2014a; 2014b; van Aert et al., 2016; Carter et al., 2019). Results so far can best be summarized by stating that the effects of QRPs can be unpredictable and complex. van Aert and colleagues even went so far as to say "In case of strong evidence or strong indications of $p$-hacking, [metaanalysts should] be reluctant in interpreting estimates of traditional meta-analytic techniques and $p$-uniform and $p$-curve because their effect-size estimates may be biased in any direction depending on the type of p-hacking used" (p.714). It is also currently unknown how QRPs affect heterogeneity estimates (Augusteijn et al., 2019).

Carter et al.'s (2019) simulations suggest that the effects of publication bias may be somewhat larger than the effects of QRPs under commonly observed conditions in the social sciences, although the generalizability of this conclusion might depend on how closely the 
conditions of their simulations do match the real world, as well as which type and combination of QRPs are used. Regardless, Carter et al.'s results need not suggest that QRPs can safely be ignored, because their simulations do show instances where QRPs are quite influential. More broadly, in real research, the effects of publication bias and QRPs will combine to bias metaanalyses in ways that make it extremely challenging to accurately account for their influences.

Selection bias and other coding issues. Meta-analysts themselves are also likely to encounter ambiguous choices and forking paths, with opportunities for flexibility in metaanalytic coding and analysis. Coding decisions for individual studies carry much ambiguity which of two dependent variables should be included in the meta-analysis? Should multiple DVs be averaged together or analyzed multivariately? Does this particular operationalization of the independent variable fit inclusion criteria? Should this study be included or excluded? Which key terms should be used, and which databases should be searched? How are unpublished studies to be located, and how is study quality to be assessed? These are only a few of the many possible decisions a meta-analyst must make.

Perhaps the largest opportunity for meta-analyst choice involves the selection and coding of meta-analytic moderators. These moderator variables are selected in order to try to explain variation (i.e., heterogeneity) in study effect sizes. They represent an opportunity to test hypotheses besides the ones that the original authors conceived. For instance, a theory might predict a stronger effect among men than women. A meta-analyst could code the participant gender ratio in existing studies to see whether samples with larger numbers of men show larger effects on average.

These choice points can morph into opportunities for QRPs when decisions are made in a data contingent way and/or based on their effects on meta-analytic conclusions. Dueling meta- 
analyses are common (see Ferguson, 2014), suggesting that it is often possible to analyze or interpret meta-analytic data in such a way as to easily support one's preconceptions or theory. Although there are methodological strategies (especially pre-registration of meta-analytic protocols) that meta-analysts can use to help protect against such biases, current practices leave ample opportunities for bias (Schmidt, 2017).

Non-cumulative methods and measurement. A meta-analyst's task is synthesizing findings on a common research question or hypothesis. Sometimes, however, the methods and measures used to investigate a question are so diverse that the resulting synthesis may lack meaning. For instance, Eysenck (1978) argued that Smith and Glass's (1977) decision to combine many very different types of psychotherapy in one analysis was problematic. Although it is possible to compute such an average, Eysenck questioned what meaning could be derived from it.

Critics like Eysenck have termed this problem "mixing apples and oranges," with the idea being that averages of such diverse "fruit" are not meaningful (Feinstein, 1995). Proponents of meta-analysis note that researchers can code methodological features of studies and include those features in the analysis, as Smith and Glass did (1977; see Chan \& Arvey, 2012). For example, studies can be classified as experimental or observational, using long-form or short-form measures, adhering to Theory A or competing Theory B, and so on. These codes then become potential moderators that might explain heterogeneity in effect sizes.

One problem is that the methodological features of various studies may end up being mostly confounded. For instance, if proponents of Theory A also tend to use long-form measures and do experiments, whereas proponents of Theory B use short-form measures and do observational studies, it becomes impossible to determine which (if any) of these ingredients is 
the critical feature that is causing a difference in observed effect size. And as noted above, metaanalytic moderators can be selected post hoc in a way that helps a meta-analyst to draw preferred conclusions. Just as in primary research, when meta-analytic moderator variables are not randomized and experimentally controlled, it becomes challenging to draw strong conclusions about the reason for an effect.

Another problem is that in some areas of research, it is possible that coding of methods or measures might reveal that many studies are "loners" or "onlys" (that is, they are the sole studies that use those methods or measurement). Author-developed or other one-off measures are very common in some research literatures. Flake, Pek, and Hehman (2017) found that nearly half of papers published in 2014 in the Journal of Personality and Social Psychology (a top journal in its subfield) were either explicitly declared to have been developed on-the-fly or failed to include references to established scales (suggesting the measures were developed ad hoc). Thus it seems that esearchers are not strongly incentivized to work cumulatively using shared paradigms.

As an example of why non-standard measurement can be problematic for research synthesis, consider Elson (2016), who has catalogued the literature on the CRTT (Competitive Reaction Time Task). The CRTT is a laboratory measure of aggressive behavior (see also Elson et al., 2014). Elson found that there were more ways to score the CRTT (156 scoring strategies) in the published literature than there were papers that used the task $(k=130)$. Interestingly, the modal scoring strategy was unique (no other study used that strategy) with 106 of the 156 strategies being used only once, ever. This is not good news for the research synthesist. A lack of cumulative research design and scoring strategies makes meta-analysis challenging at best, and in some cases, it may render meaningful summaries very difficult.

\section{Alternatives to and Supplements for Meta-Analysis}


Given the potential severity of the problems just outlined for meta-analytic conclusions, one might speculate as to whether there are better alternatives to traditional meta-analysis when seeking to synthesize research conclusions. This section considers possible alternatives to, and supplements for, meta-analysis as currently practiced in the social sciences.

Narrative review. Meta-analytic (or quantitative) reviews can be contrasted with traditional narrative reviews, often simply called literature reviews. Generally, in a narrative review, an author gives an overview of relevant previous work that provides context and justification for a subsequent study or future line of research. These reviews are usually not meant to be comprehensive, but rather they summarize particular features of a research literature (Grant \& Booth, 2009). Narrative reviews also do not contain a quantitative synthesis of research evidence.

It is possible that a research community could consider abandoning meta-analyses altogether and return to doing narrative reviews only. But the observant reader might recall that meta-analyses were invented because narrative reviews were found wanting (Borenstein et al., 2009; see also Meehl, 1990). Indeed, narrative reviews are not well-suited to summarizing large bodies of evidence, and unlike meta-analysis, there is no explicit weighting of different studies.

Narrative reviews might even encourage a "vote-counting" method in which researchers simply tally up the studies "for" or "against" a hypothesis. Such methods are flawed (Gurevitch et al., 2018), because, among other reasons, they fail to take the precision of estimates into account, implicitly treating all studies as equal.

Another issue is cherry picking. In a narrative review, there is nothing preventing authors from simply presenting only those studies that are supportive of their positions and viewpoints (even unintentionally). For all of these reasons, it seems unlikely that researchers will view 
narrative review as a viable alternative to meta-analysis.

Systematic review. Systematic review is a method for transparently conducting rigorous and comprehensive literature reviews (Grant \& Booth, 2009). Importantly, systematic reviews are not just high quality qualitative reviews. The term systematic review includes a method for reviewing research literature that should produce reproducible and unbiased findings. Systematic reviews are likewise not solely quantitative reviews; they can include meta-analyses but are not required to do so (Haddaway \& Bilotta, 2014).

There are several key ingredients that distinguish systematic reviews from traditional narrative reviews (Haddaway, Land, \& Macura, 2017). First, systematic reviews must use transparent and reproducible methods to locate relevant studies, and these methods must be described in sufficient detail to allow reproducibility. Best practices include searching multiple databases and naming those databases in the paper, clearly describing strategies used to locate unpublished works, and providing the exact search strings used (in the paper or a supplement).

Second, systematic reviews must have systematic procedures for evaluating studies both published and unpublished (i.e., grey literature) - for inclusion or exclusion in the review. Researchers first screen titles and abstracts, eliminating studies that obviously don't fit inclusion criteria. The remaining studies are evaluated to ensure they meet inclusion criteria. As a best practice, researchers should have independent coders assess each study for inclusion (and measure interrater reliability), and they must systematically track decisions to include or exclude studies.

Finally, systematic reviews must include critical appraisal, also known as risk of bias assessment or quality assessment, of the constituent studies in the review. Critical appraisal means that studies are evaluated to ensure that they possess certain quality or design features. 
Many different critical appraisal systems exist; there is not one gold-standard method (Sanderson et al., 2007). The GRADE system (Guyatt et al., 2008) provides a further scheme for evaluating the quality of evidence and strength of recommendations in whole literatures rather than individual studies.

Additionally, some bodies (The Cochrane Collaboration; Campbell Collaboration; Center for Environmental Evidence [CEE]) require publication of a peer-reviewed protocol prior to the commencement of a systematic review. A protocol serves as a pre-registration for the review, which can help to constrain reviewers' researcher degrees of freedom and prevent bias in the review. The Campbell Collaboration accepts reviews that are relevant to social science disciplines, but the reviews need to have a focus on policy applications. There is no currently existing home for peer-reviewed registrations of systematic reviews for basic social science research.

Another related type of review is called a systematic map (James et al., 2015), also known as an evidence map or gap map. Systematic maps use systematic procedures to locate studies on a topic, but they do not synthesize the evidence from these studies (e.g., using metaanalysis), nor do they employ formal critical appraisal. What the maps can do is reveal areas or topics that have been understudied and are therefore missing information. In principle, it would be possible for researchers to evaluate the studies gathered in a systematic map and use this evaluation to support new, pre-registered data collection. For instance, if studies showed either a limited evidence base or substantial evidence of questionable research practices or other biases, researchers could opt not to convert a systematic map to a systematic review (with metaanalysis). Instead, they could simply collect new (less biased) data.

Systematic reviews have become somewhat popular in biomedicine (Bastian, Glasziou, \& 
Chalmers, 2010), but uptake in the social sciences has been slower. Since 1980, Psychological Bulletin, a premier outlet for review papers in psychology, has published 246 (13.4\%) original research papers (i.e., not commentaries, retractions, corrections, or editorials) with "metaanalysis" in their titles ${ }^{3}$, but just $12(0.6 \%)$ papers with "systematic review" (out of 1,833 original research papers). Of course, a lack of consistency in terminology means that some metaanalyses in psychology might actually be adhering to many or most of the methodological protections that are present in systematic reviews, but they are not being labeled as such. It could also be that some papers with the label systematic review are not truly systematic reviews. Haddaway, Land, and Macura (2017) find that misunderstandings of the term systematic review are common in their field (environmental ecology and conservation), a finding that likely extends to the social sciences (see also Petticrew, 2001).

Even if many meta-analyses were already taking great care with search reproducibility and tracking study exclusion (and there is evidence they do not; see Moher et al., 2007), most psychology meta-analyses are missing a formal critical appraisal step (Aytug et al., 2012; Bastian, 2016), rendering them ineligible to be considered true systematic reviews. Again, critical appraisal involves the systematic evaluation of study quality using a tool such as the Cochrane Collaboration's checklist (Higgins et al., 2011). Furthermore, only a handful (e.g., Jones et al., 2018; Woll \& Schönbrodt, in press) have ever been pre-registered ${ }^{4}$, and there is no central collaboration akin to Cochrane, Campbell, or CEE in psychology. Taken together, the evidence suggests that although psychology and related social sciences make frequent use of

\footnotetext{
${ }^{3}$ Including the phrase "meta-analysis" or "systematic review" in the title is a best practice, but not one that is always followed. Hand searching of volumes of Psychological Bulletin would likely reveal both more meta-analyses and more systematic reviews.

${ }^{4}$ Reviews with health-related outcomes are eligible for registration in PROSPERO, and some studies have been registered there.
} 
meta-analyses, these meta-analyses are not often of the high methodological rigor required for the use of the label systematic review.

In sum, systematic reviews hold some promise in terms of their ability to increase quality and decrease bias in research syntheses. Moher et al. (2007) report that Cochrane reviews, which have very rigorous standards for peer-review, pre-registration, execution, and reporting, are of demonstrably higher quality than non-Cochrane reviews. Moher et al.'s findings are an existence proof that it is therefore possible for a field to make its meta-analyses more rigorous.

Systematic reviews are unlikely to be a panacea for several reasons. First, low quality and high risk of bias in existing studies may mean that even the most rigorously conducted reviews are ultimately uninformative (at least in terms of yielding an interpretable summary effect size). Second, full systematic reviews are laborious and take even more time than non-systematic reviews to complete. Critics might correctly wonder whether the quality vs. time-to-completion trade-off is worth it. Finally, even Moher et al.'s (2007) evidence shows that systematic review methodology (as employed in Cochrane reviews) still shows a lot of room for improvement. Thus, although pre-registration of meta-analyses and coordination by a collaborative body stands to improve methods and reporting substantially, systematic review cannot fully solve the problems of publication bias and poor quality of evidence in the literature.

Bias-corrected meta-analysis. The preceding sections have described how biases in individual study estimates (questionable research practices) and in whole literatures (publication bias) can affect meta-analytic conclusions. Perhaps one way to address these biases is simply to take account of them and apply corrections. This logic undergirds the many bias correction tools that are available to meta-analysts. I consider the rationale, as well as strengths and weaknesses of the most often used bias correction tools. 


\section{Popular, but poorly performing, methods of bias correction: Fail-safe $N$ and trim and}

fill. Fail-safe $N$ should more realistically be thought of as a bias detector rather than a bias corrector, but I consider it here because of its widespread use. Importantly, even as a bias detector, fail-safe $N$ underperforms relative to alternatives, and it should realistically not be used at all.

Rosenthal (1979) proposed fail-safe $N$ as a way to assess how many results with effect size equal to zero would be needed to nullify a statistically significant meta-analysis. Although computationally simple, Becker (2005) reviews the many problems with this statistic, noting (among other things) the lack of a formal statistical model supporting its use and the lack of a clear criterion for identifying large test values. Furthermore, Becker (2005) notes that the statistic assumes that missing studies are exclusively null, as opposed to negative (Begg \& Berlin, 1988; Ferguson \& Heene, 2012).

In practice, fail-safe $N$ s are often large, implying that huge numbers of studies would be needed to change a meta-analytic conclusion. Cases like ego depletion (discussed previously) suggest that fail-safe $N$ offers an overly optimistic take on the problem of publication bias (Hilgard, 2016). Given this lack of validity together with the aforementioned statistical critiques, researchers are well-served to avoid using fail-safe $N$.

Trim and fill (Duval \& Tweedie, 2000) is a test that relies on funnel plot asymmetry to detect and correct publication bias. A funnel plot is a graphic that plots study effect sizes (on the $\mathrm{x}$-axis) against the inverse of their precisions (on the y-axis). In unbiased literatures, this results in a symmetric plot, with more precisely estimated studies closer to the average overall effect size, with less precisely estimated studies tending to show more variable effect sizes, symmetrically in the positive and negative direction. In biased literatures, funnel plots show 
asymmetry; there is a negative correlation between study precision and effect size. (It should be noted that selection bias is not the only cause of funnel plot asymmetry, but it is one major cause.)

In a trim and fill analysis, outlying studies are iteratively removed ("trimmed") from one side of the funnel plot until the plot is symmetric, and the new axis of symmetry is taken as an adjusted estimate of the effect size. The resulting estimate has a variance that is too small, so the studies that were removed are added back and additional studies are then imputed ("filled") to again achieve symmetry (Borenstein et al., 2009).

Moreno et al. (2009) compared trim and fill to various forms of meta-regression (discussed subsequently). They found that trim and fill consistently underperformed relative to these alternative models, especially when there was heterogeneity in effect sizes. Carter et al.'s (2019) simulation agreed, finding that trim and fill gave positively biased effect sizes under publication bias, in addition to having very high type I error rates.

These authors and others (e.g., Simonsohn et al., 2014a; McShane et al., 2016; van Assen et al., 2015) converge in their recommendation to avoid the use of trim and fill as a bias correction technique. As a bias detector, there are other tools that will do a better job, but researchers can consider trim and fill as one of several tools in a sensitivity analysis (Carter et al., 2019).

\section{Selection models: p-curve, p-uniform, and three-parameter selection model (3PSM).}

Selection models are another class of bias correction tools. Overall, these models assume that the published literature represents a subset of studies that have been performed, and the analysis attempts to fit parameters that model this selective publication process. Different selection models make differing assumptions about patterns of publication bias. They then use these 
assumptions to fit a model that corrects for missing studies.

The simplest of these models assumes that the cutoff for statistical significance (i.e., $p<$ .05) fully determines which results will be published/observed. That is, non-statistically significant results are not included in the meta-analysis. These models also assume that all of the studies in an analysis measure the same population effect size (that is, there is no heterogeneity beyond sampling error). The model then estimates one parameter, $\mu$, which is the average effect size adjusting for studies missing due to publication bias. Hence, these models are known as oneparameter selection models (McShane et al., 2016).

After an early introduction (Hedges, 1984), these models have had renewed interest under slightly different specifications under the names $p$-curve (Simonsohn et al., 2014b) and $p$ uniform (van Assen et al., 2015). The basic logic behind these models is that $p$-values are uniformly distributed under the null hypothesis, whereas under non-null models, the distribution of $p$-values varies based on statistical power. As power increases, the proportion of small $p$ values $(p<.01)$ observed also increases. Therefore, for literatures investigating true effects, small $p$-values should frequently be observed, whereas $p$-values near the .05 cutoff should be relatively rare.

In terms of strengths, $p$-curve and $p$-uniform do a good job of recovering the true effect size when model assumptions are met (i.e., when there is no heterogeneity ${ }^{5}$, when $p<.05$ is a firm selection criterion, and all results with $p<.05$ are equally publishable). They are relatively simple to implement, though researchers frequently make mistakes regarding which $p$-values to select (Nelson, Simmons, \& Simonsohn, 2017). However, both $p$-curve and $p$-uniform perform

\footnotetext{
${ }^{5}$ Simmons, Nelson, \& Simonsohn (2018; http://datacolada.org/67) disagree that $p$-curve performs poorly when there is effect size heterogeneity. They claim, instead, that $p$-curve accurately recovers the average true effect size "of the studies included in $p$-curve." This effect size is in contrast to the population effect size that meta-analysis typically attempts to recover.
} 
quite poorly when there is heterogeneity in effect sizes (van Aert et al., 2016; Carter et al., 2019). Additionally, $p$-hacking can lead to either under- (Simonsohn et al., 2014a; 2014b) or over- (van Aert et al., 2016; Bruns \& Ioannidis, 2016; Ulrich \& Miller, 2015) estimation of corrected effect sizes by $p$-curve and $p$-uniform. van Aert et al. note that " $p$-hacking may bias $p$-uniform and $p$ curve estimates in any direction depending on the type of $p$-hacking and (b) $p$-uniform and $p$ curve estimates are not necessarily better than those of fixed effect meta-analysis when $p$ hacking occurs" (p. 721).

These issues have led van Aert et al. (2016) to recommend avoiding meta-analytic synthesis when there is strong evidence of $p$-hacking. They also recommend using subgroups to try to achieve a homogeneous subset of studies for analysis, addressing the heterogeneity issue. In reality, identifying the appropriate subgroups for analysis is a non-trivial task as the variable(s) needed to mark subgroups may be unknown or unmeasured. Another issue with these models involves selecting the appropriate hypothesis tests for inclusion in the analysis (Simonsohn et al., 2014). The method does not handle dependent $p$-values (e.g., tests from related dependent variables taken from the same sample), so meta-analysts must specify a plan ahead of time to help them choose which of the many $p$-values in a paper to include in the analysis. Neither $p$-curve nor $p$-uniform can presently test moderator variables.

More complex selection models exist, such as Iyengar and Greenhouse's (1988) threeparameter selection model (3PSM; see also Hedges \& Vevea, 2005). This model has a mean parameter (like $p$-curve and $p$-uniform) in addition to a heterogeneity parameter and a selection parameter (thus yielding the eponymous three parameters). The selection parameter applies weights that account for publication bias. In contrast to $p$-curve and $p$-uniform, which ignore studies with $p>.05$ completely, the 3PSM takes non-significant studies into account using a 
weight function.

On the plus side, the 3PSM has more realistic assumptions behind it than one parameter models (given that publication bias is unlikely to be acting precisely at the .05 cutoff, and heterogeneity is likely to be present in most literatures). Furthermore, the model can be flexibly extended to incorporate formal tests of moderation without the need to rely on sub-group analysis. On the minus side, these techniques are complex enough that they may be difficult to implement for non-specialists (though a recently developed R package, weightr [Coburn \& Vevea, 2018], makes the model more accessible though the package makes assumptions to yield higher convergence rates). Additionally, model convergence may be an issue, particularly when the number of studies in a meta-analysis is small. Further, the weight function, even if estimated, may not be well recovered by the model (Hedges \& Vevea, 2005). Crucially, the performance of the model depends on the accuracy of the selection function, which is to some extent unknowable (Augusteijn et al., 2019).

Carter et al. (2019) report that the 3PSM showed reasonable type I error rates but was underpowered under many simulated conditions that match those often observed in the social sciences. In short, $p$-curve and $p$-uniform rely on some important assumptions and method limitations that may render them less useful for meta-analysis than one might hope. The 3PSM also suffers from some important limitations, most crucially relying on a large number of studies to reliably converge (unless strict assumptions are allowed). Investigations into these models under various conditions remain ongoing, rendering firm conclusions unwarranted.

Meta-regression: Egger's test and PET-PEESE. A final set of bias correction models again rely on the logic of a funnel plot to examine publication bias. These tools, broadly known as meta-regression techniques, involve using regression to model the association between effect 
sizes and study precision. Egger, Smith, Schneider, and Minder (1997) proposed regressing logged odds ratios on study precisions, with statistically significant slope coefficients indicating funnel plot asymmetry and therefore publication bias.

Stanley and Doucouliagos (2014) extended Egger et al.’s (1997) logic to examining not only the slope of this regression, but also using the model intercept as an estimate of metaanalytic effect size after correcting for publication bias. They called this test PET (Precision Effect Test). Another variant of this model uses study variances (i.e., standard error squared) rather than standard errors to predict study effect sizes, yielding a quadratic model called PEESE (Precision Effect Estimate with Standard Error). Stanley and Doucouliagos (2014) recommend using PET-PEESE as a conditional test in which the analyst proceeds to run a PEESE model only after rejecting the null hypothesis of no effect in a PET model. This conditional test was chosen due to the fact that PET is biased downward when the null hypothesis is false, but PEESE is biased upward when the null hypothesis is true. However, PET may be underpowered in many common research scenarios, leading some to recommend that analysts avoid using the conditional test (Hilgard, 2017).

Like the 3PSM, meta-regression models have the possibility to include and test moderating variables, making them more flexible than $p$-curve or $p$-uniform. PET-PEESE also showed fairly good type I error rates, but it had low power under many conditions (Carter et al., 2019). Stanley (2017) reviews additional potential problems with PET-PEESE as applied under common scenarios in social science research literatures. Specifically, when all individual studies are underpowered, PET and PEESE do not accurately recover effect sizes. Furthermore, because they are regression models, a large number of studies are needed for the models to perform as intended (though convergence was not as much of an issue as it was for the 3PSM). Thus, 
Stanley's $(2017)^{6}$ results suggest that small scale meta-analyses may be inappropriate for metaregression techniques. Finally, PET and PEESE may be underpowered relative to naïve (i.e., uncorrected) fixed effects and random effects meta-analysis, especially when there is little to no publication bias in reality.

Summary. Meta-analysts are faced with many choices when it comes to selecting a technique to correct for the biasing effects of publication bias and questionable research practices. Several factors are important to consider when choosing a test, including: (1) the size of a typical study (2) the number of studies, $k$, (3) variance in sample sizes, (4) likely severity of publication bias, (5) severity and type of $p$-hacking, and (6) effect size heterogeneity. Carter et al. (2019) offer a web application, metaExplorer, which researchers can use to assess when a particular technique may or may not be appropriate for their review. Augusteijn et al. (2019) also offer Qsense, a web application that can help researchers understand how heterogeneity estimates may be biased under varying conditions.

Note, however, that many of these factors that researchers must take into account to choose a bias correction tool are precisely the factors that they would like for meta-analyses to estimate (e.g., degree of heterogeneity)! Thus, researchers are left in an unsettling place. Although it would be ideal if applying bias correction tools led to straightforward recovery of the unbiased meta-analytic effect size, the various simulation studies reviewed here suggest that they are unlikely to do so. Yet, uncorrected (naïve) meta-analysis often performs far worse than these bias corrected tools. When investigating a null effect under conditions of heavy publication bias (perhaps an all too frequent occurrence in social science research), random effects meta-analysis

\footnotetext{
${ }^{6}$ Readers should note that another technique known as Top10 (Stanley, Jarrell, \& Doucouliagos, 2010) proposes to reduce the influence of likely biased small studies by meta-analyzing only the top $10 \%$ most precisely estimated studies. Given that many social science meta-analyses involve small numbers of studies, this technique may not be viable under common circumstances; it remains to be seen whether Top 10 proves to be useful for bias-correction.
} 
nearly always yields a wrong answer. In brief, the best researchers can do is to apply a variety of corrections and assess the sensitivity of their conclusions to the models applied (Carter et al., 2019; Applebaum et al., 2018). Easy solutions are not forthcoming.

\section{Large scale pre-registered trials, registered reports, and registered multi-lab}

studies. Bias correction tools implicitly assume that the magnitude of bias in a research literature is (1) discoverable and (2) correctable. To the extent that these assumptions are unsupported, researchers may be better off collecting new data on a question, rather than trying to extract meaning from the existing literature. Two possibilities exist to support the collection and analysis of less biased data: unreviewed preregistration and registered reports.

Pre-registration implies the full specification of study design and data analysis prior to commencing a study, and it may also (optionally) involve specification of a priori, theoryderived hypotheses. Unreviewed pre-registrations are generated by researchers themselves and submitted to a registry before data collection begins. The pre-registration is then later linked in the final journal article, where peer reviewers and later readers can compare the pre-registration plan to the ultimately published paper.

By contrast, a registered report involves editor-supervised peer review of a pre-registered study proposal at a participating journal, again prior to the onset of data collection (Chambers, 2013). Aside from peer review of study proposals (termed stage one protocols), a unique feature of registered reports is in principle acceptance (IPA). IPA means that participating journals agree to publish accepted registered reports regardless of the statistical significance of the key hypothesis test. Instead, publication decisions are made based on a priori defined conditions, such as whether a study passes certain quality thresholds (termed positive controls).

Registered replication reports (RRRs) are another recent innovation, pioneered at the 
journal Perspectives in Psychological Science (Simons, Holcombe, \& Spellman, 2014) and now rehomed in the newly formed journal Advances in Methods and Practices in Psychological Science (Simons, 2018). RRRs are a form of registered report that focus on direct replications (i.e., studies that attempt to duplicate as many features of an original study as possible), involve adversarial collaboration (i.e., input from both proponents and skeptics of an effect), and have data collection distributed over many participating laboratories. It should be noted that multi-lab registered reports can also involve novel research, not just replication studies (Schweinsberg et al., 2016).

Well powered pre-registered studies - perhaps especially those run through the registered reports mechanism - may ultimately be more informative than retrospective meta-analyses. In particular, if researchers want to be able to manipulate and control potential moderators, collecting new data may well be the best mechanism to do so (because researchers can avoid the uncontrolled nature of moderators in existing studies, as well as potential confounding of sitelevel variables). Furthermore, pre-registered multi-site studies allow researchers to assess the magnitude of heterogeneity under conditions that are known to have limited researcher degrees of freedom and be free of publication bias.

However, well-done large trials are extremely resource intensive, and the decision to pursue one likely needs to be informed by existing evidence. Thus, researchers might find themselves in the tricky position of needing to complete a systematic map or a full systematic review (and perhaps meta-analysis) before deciding whether or not to run a large scale registered replication. Ultimately the decision to pursue a review rather than collect new data will depend on idiosyncratic features of the research question at hand, but researchers should recognize that a directive to simply avoid meta-analysis in favor of new data collection is unrealistic. 


\section{Conclusion and Recommendations for Informative and Reproducible Meta-Analyses}

In the forty years since their modern introduction, meta-analyses have gone from being viewed as mega-silliness (Eysenck, 1978) and statistical alchemy (Feinstein, 1995), to being regarded as a precipitator of Kuhnian revolutions (Chan \& Arvey, 2012) and "platinum" evidence (Stenenga, 2011), to once again being regarded with suspicion (Engber, 2016). What this review has hopefully shown is that core issues - related to publication bias, meta-analytic coding decisions, and non-cumulative methods and measurement - as well as newly recognized issues, like questionable research practices, are serious challenges to the validity of metaanalysis. Furthermore, no single suggested change or improvement (abandoning synthesis altogether, switching to systematic reviews, employing bias correction tools, switching to preregistered trials only) is likely to be sufficient to address these problems.

In combination, these tools would have the chance to improve the quality of metaanalytic synthesis. Lakens, Staaks, and Hilgard (2016) offer six further suggestions for making meta-analyses more reproducible, and readers that are considering undertaking a meta-analysis or systematic review are advised to carefully attend to each of their points.

It may well be the case that spending fewer resources on research synthesis and more resources on improving the quality (and reducing the bias) in the published literature may be a more productive use of researchers' limited time. Yet, the question remains as to how we decide which areas of the literature to invest more resources in and which to abandon. Increasing the quality of meta-analytic synthesis using the strategies described above, as well as working collaboratively and using multi-lab methodologies to determine how to move forward (Moshontz et al., 2018), seems to be our only option. Easy solutions are not forthcoming. 


\section{References}

Appelbaum, M., Cooper, H., Kline, R. B., Mayo-Wilson, E., Nezu, A. M., \& Rao, S. M. (2018). Journal article reporting standards for quantitative research in psychology: The APA Publications and Communications Board task force report. American Psychologist, 73(1), $3-25$.

Augusteijn, H.E.M., van Aert, R.C.M., \& van Assen,M.A.L.M. (2019). The effect of publication bias on the assessment of heterogeneity. Psychological Methods, 24, 116-134.

Aytug, Z. G., Rothstein, H. R., Zhou, W., \& Kern, M. C. ( 2012). Revealed or Concealed? Transparency of procedures, decisions, and judgment calls in meta-analyses. Organizational Research Methods, 15, 103-133.

Bakker, M., van Dijk, A., \& Wicherts, J. M. (2012). The rules of the game called psychological science. Perspectives on Psychological Science, 7, 543-554.

Bastian, H. (2016, July 5). Psychology’s meta-analysis problem. Retrieved from http://blogs.plos.org/absolutely-maybe/2016/07/05/psychologys-meta-analysis-problem/

Bastian, H., Glasziou, P., \& Chalmers, I. (2010). Seventy-five trials and eleven systematic reviews a day: How will we ever keep up? PLoS Medicine, 7(9), e1000326. doi: 10.1371/journal.pmed.1000326

Becker, B. J. (2005). Failsafe $N$ or file-drawer number. In H. R. Rothstein, A. J. Sutton, \& M. Borenstein (Eds.) Publication bias in meta-analysis - prevention, assessment, and adjustments (pp. 111-125) Chichester, UK: John Wiley \& Sons, Inc.

Begg, C.B. and Berlin, J.A. (1988). Publication bias: A problem in interpreting medical data (with discussion). Journal of the Royal Statistical Society, Series A, 151, 419-463.

Borenstein, M., Hedges, L. V., Higgins, J. P., \& Rothstein, H. R. (2011). Introduction to meta- 
analysis. Chichester, UK: John Wiley \& Sons.

Borgerson, K. (2009). Valuing evidence: bias and the evidence hierarchy of evidence-based medicine. Perspectives in Biology and Medicine, 52(2), 218-233.

Bruns, S. B., \& Ioannidis, J. P. (2016). p-curve and $p$-hacking in observational research. PLoS One, 11(2), e0149144.

Carter, E. C., \& McCullough, M. E. (2014). Publication bias and the limited strength model of self-control: Has the evidence for ego depletion been overestimated? Frontiers in Psychology, 5, 823. doi: 10.3389/fpsyg.2014.00823

Carter, E. C., Schönbrodt, F. D., Gervais, W. M., \& Hilgard, J. (2019). Correcting for bias in psychology: A comparison of meta-analytic methods. Advances in Methods and Practices in Psychological Science, 2(2), 115-144.

Chalmers, I. Hedges, L. V., \& Cooper, H. (2002). A brief history of research synthesis. Evaluation and the Health Professions, 25, 12-37.

Chan, M. E., \& Arvey, R. D. (2012). Meta-analysis and the development of knowledge. Perspectives on Psychological Science, 7, 79-92.

Coburn, K. M., \& Vevea, J. L. (2018). weightr: Estimating weight-function models for publication bias in r. R package version 1.1.2. Retrieved from https://CRAN.R-project. org/package=weightr

Doi, S. A., \& Thalib, L. (2008). A quality-effects model for meta-analysis. Epidemiology, 19, 94100.

Duval, S., \& Tweedie, R. (2000). Trim and fill: a simple funnel-plot-based method of testing and adjusting for publication bias in meta-analysis. Biometrics, 56, 455-463.

Egger, M., Smith, G. D., Schneider, M., \& Minder, C. (1997). Bias in meta-analysis detected by 
a simple graphical test. $B M J, 315,629-634$.

Elson, M. (2016). FlexibleMeasures.com: Competitive Reaction Time Task. Retrieved from http://www.flexiblemeasures.com/crtt/ doi:10.17605/OSF.IO/4G7FV

Elson, M., Mohseni, M. R., Breuer, J., Scharkow, M., \&amp; Quandt, T. (2014). Press CRTT to measure aggressive behavior: The unstandardized use of the Competitive Reaction Time Task in aggression research. Psychological Assessment, 26, 419-432. doi: $10.1037 / \mathrm{a} 0035569$

Engber, D. (2016, March 6). Everything is crumbling. Retrieved from http://www.slate.com/articles/health_and_science/cover_story/2016/03/ego_depletion_an _influential_theory_in_psychology_may_have_just_been_debunked.html

Eysenck, H. J. (1978). An exercise in mega-silliness. American Psychologist, 33, 517.

Fanelli, D. (2010). "Positive" results increase down the hierarchy of the sciences. PloS One, 5, e10068.

Feinstein, A. R. (1995). Meta-analysis: Statistical alchemy for the 21st century. Journal of Clinical Epidemiology, 48, 71-79.

Ferguson, C. J., \& Heene, M. (2012). A vast graveyard of undead theories: Publication bias and psychological science's aversion to the null. Perspectives on Psychological Science, 7, $555-561$.

Ferguson, C. J. (2014). Comment: Why meta-analyses rarely resolve ideological debates. Emotion Review, 6, 251-252.

Fisher, R. A. (1932). Statistical methods for research workers (4th ed.). London: Oliver and Boyd.

Flake, J. K., Pek, J., \& Hehman, E. (2017). Construct validation in social and personality 
research: Current practice and recommendations. Social Psychological and Personality Science, 8, 370-378.

Franco, A., Malhotra, N., \& Simonovits, G. (2014). Publication bias in the social sciences: Unlocking the file drawer. Science, 345, 1502-1505.

Friese, M., Loschelder, D. D., Gieseler, K., Frankenbach, J., \& Inzlicht, M. (2019). Is ego depletion real? An analysis of arguments. Personality and Social Psychology Review, 23, 107-131. doi: $10.1177 / 1088868318762183$

Gelman, A., \& Loken, E. (2013). The garden of forking paths: Why multiple comparisons can be a problem even when there is no "fishing expectation" or "p-hacking" and the research hypothesis was posited ahead of time. Retrieved from http://www.stat.columbia.edu/ gelman/research/ unpublished/p_hacking.pdf

Glass, G. V. (2015). Meta-analysis at middle age: A personal history. Research Synthesis Methods, 6, 221-231.

Grant, M. J., \& Booth, A. (2009). A typology of reviews: an analysis of 14 review types and associated methodologies. Health Information \& Libraries Journal, 26, 91-108.

Gurevitch, J., Koricheva, J., Nakagawa, S., \& Stewart, G. (2018). Meta-analysis and the science of research synthesis. Nature, 555, 175-182.

Guyatt, G. H., Oxman, A. D., Vist, G. E., Kunz, R., Falck-Ytter, Y., Alonso-Coello, P., \& Schünemann, H. J. (2008). GRADE: An emerging consensus on rating quality of evidence and strength of recommendations. BMJ, 336, 924-926.

Haddaway, N. R., \& Bilotta, G. S. (2016). Systematic reviews: Separating fact from fiction. Environment International, 92, 578-584.

Haddaway, N. R., Land, M., \& Macura, B. (2017). “A little learning is a dangerous thing”: A call 
for better understanding of the term 'systematic review'. Environment International, 99, 356-360.

Hagger, M. S., Wood, C., Stiff, C., \& Chatzisarantis, N. L. D. (2010). Ego depletion and the strength model of self-control: A meta-analysis. Psychological Bulletin, 136, 495-525.

Hagger, M. S., Chatzisarantis, N. L. D., Alberts, H., Anggono, C. O., Batailler, C., Birt, A. R., ... \& Zweinberg, M. (2016). A multilab preregistered replication of the ego-depletion effect. Perspectives on Psychological Science, 11, 546-573.

Hedges, L. V. (1984). Estimation of effect size under nonrandom sampling: The effects of censoring studies yielding statistically insignificant mean differences. Journal of Educational Statistics, 9, 61-85.

Hedges, L. V., \& Vevea, J. (2005). Selection method approaches. In H. R. Rothstein, A. J. Sutton, \& M. Borenstein (Eds.) Publication bias in meta-analysis - prevention, assessment, and adjustments (pp. 145-174) Chichester, UK: John Wiley \& Sons, Inc.

Higgins J. P.T., Altman, D. G., Gøtzsche, P. C., Jüni, P., Moher, D., Oxman, A. D., Savovic, J., Schulz, K. F., Weeks, L., \& Sterne, J. A. C. (2011). The Cochrane Collaboration's tool for assessing risk of bias in randomised trials. $B M J, 343, \mathrm{~d} 5928$.

Hilgard, J. (2016, July 19). The failure of fail-safe N. Retrieved from http://crystalprisonzone.blogspot.com/2016/07/the-failure-of-fail-safe-n.html Hilgard, J. (2017). Response to Colada [59]. Retrieved from http://datacolada.org/wpcontent/uploads/2017/04/Response-by-Joe-Hilgard-to-Colada-59.pdf

Hunt, M. (1997). How science takes stock: The story of meta-analysis. Russell Sage Foundation. Ioannidis, J. P. (2005). Why most published research findings are false. PLoS medicine, 2(8), e124. 
Iyengar, S., \& Greenhouse, J. B. (1988). Selection models and the file drawer problem. Statistical Science, 3, 109-117.

James, K. L., Randall, N. P., \& Haddaway, N. R. (2015). A methodology for systematic mapping in environmental sciences. Environmental Evidence, 5. doi:10.1186/s13750-0059-6

John, L. K., Loewenstein, G., \& Prelec, D. (2012). Measuring the prevalence of questionable research practices with incentives for truth telling. Psychological Science, 23, 524-532.

Jones, A., Remmerswaal, D., Verveer, I., Robinson, E., Franken, I. H., Wen, C. K., \& Field, M. (2018, May 29). Compliance with ecological momentary assessment protocols in substance users: A meta-analysis. Retrieved from https://doi.org/10.31234/osf.io/k4agy

Kerr, N. L. (1998). HARKing: Hypothesizing after the results are known. Personality and Social Psychology Review, 2, 196-217.

L'Abbé, K. A., Detsky, A. S., \& O'Rourke, K. (1987). Meta-analysis in clinical research. Annals of Internal Medicine, 107(2), 224-233.

Lakens, D., Hilgard, J., \& Staaks, J. (2016). On the reproducibility of meta-analyses: Six practical recommendations. BMC Psychology, 4(1), 24.

Mantel N., \& Haenszel, W. (1959). Statistical aspects of the analysis of data from the retrospective analysis of disease. Journal of the National Cancer Institute, 22, 719-748.

McShane, B. B., Böckenholt, U., \& Hansen, K. T. (2016). Adjusting for publication bias in metaanalysis: An evaluation of selection methods and some cautionary notes. Perspectives in Psychological Science, 11, 730-749.

Meehl, P. E. (1990). Why summaries of research on psychological theories are often uninterpretable. Psychological Reports, 66, 195-244.

Moher, D., Tetzlaff, J., Tricco, A. C., Sampson, M., \& Altman, D. G. (2007). Epidemiology and 
reporting characteristics of systematic reviews. PLoS Medicine, 4, e78. doi:

10.1371/journal.pmed.0040078

Moreno, S. G., Sutton, A. J., Ades, A. E., Stanley, T. D., Abrams, K. R., Peters, J. L., \& Cooper, N. J. (2009). Assessment of regression-based methods to adjust for publication bias through a comprehensive simulation study. BMC Medical Research Methodology, 9:2. doi: $10.1186 / 1471-2288-9-2$

Moshontz, H., Campbell, L., Ebersole, C. R., IJzerman, H., Urry, H. L., Forscher, P. S., ... \& Flake, J. K. (2018). Psychological Science Accelerator: Advancing Psychology through a Distributed Collaborative Network. Advances in Methods and Practices in Psychological Science, 1, 501-515.

Nelson, L. D., Simmons, J. P., \& Simonsohn, U. (2017, May 8). Forthcoming in JPSP: A nondiagnostic audit of psychological research. Retrieved from http://datacolada.org/60

O’Boyle Jr., E. H., Banks, G. C., \& Gonzalez-Mulé, E. (2017). The chrysalis effect: How ugly initial results metamorphosize into beautiful articles. Journal of Management, 43(2), 376399.

Open Science Collaboration. (2015). Estimating the reproducibility of psychological science. Science, 349(6251), aac4716.

Pearson, K. (1904). Antityphoid inoculation. British Medical Journal, 2, 1667-1668.

Petticrew, M. (2001). Systematic reviews from astronomy to zoology: myths and misconceptions. BMJ, 322(7278), 98-101.

Rosenthal, R. (1979). The file drawer problem and tolerance for null results. Psychological Bulletin, 86, 638-641.

Rosenthal, R. (2015). Reflections on the origins of meta-analysis. Research Synthesis Methods, 
$6,240-245$.

Sanderson, S., Tatt, I. D., \& Higgins, J. P. T. (2007). Tools for assessing quality and susceptibility to bias in observational studies in epidemiology: A systematic review and annotated bibliography. International Journal of Epidemiology, 36, 666-676.

Scherer R. W., Langenberg, P., \& von Elm, E. (2007). Full publication of results initially presented in abstracts. Cochrane Database of Systematic Reviews, 2, MR000005. DOI: 10.1002/14651858.MR000005.pub3

Schmidt, F. L. (2017). Statistical and measurement pitfalls in the use of meta-regression in metaanalysis. Career Development International, 22, 469-476.

Schmidt, F. L. (2015). History and introduction to the Schmidt-Hunter meta-analysis methods. Research Synthesis Methods, 6, 232-239.

Schweinsberg, M., Madan, N., Vianello, M., Sommer, S. A., Jordan, J., Tierney, W., ... \& Srinivasan, M. (2016). The pipeline project: Pre-publication independent replications of a single laboratory's research pipeline. Journal of Experimental Social Psychology, 66, 5567.

Simmons, J. P., Nelson, L. D., \& Simonsohn, U. (2011). False-positive psychology: Undisclosed flexibility in data collection and analysis allows presenting anything as significant. Psychological Science, 22, 1359-1366.

Simmons, J. P., Nelson, L. D., \& Simonsohn, U. (2018, January 8). p-curve handles heterogeneity just fine. Retrieved from http://datacolada.org/67

Simons, D. J. (2018). Introducing Advances in Methods and Practices in Psychological Science. Advances in Methods and Practices in Psychological Science, 1, 3-6.

Simons, D. J., Holcombe, A. O., \& Spellman, B. A. (2014). An introduction to Registered 
Replication Reports at Perspectives on Psychological Science. Perspectives on Psychological Science, 9, 552-555. doi:10.1177/1745691614543974

Simonsohn, U., Nelson, L. D., \& Simmons, J. P. (2014a). p-Curve and effect size: Correcting for publication bias using only significant results. Perspectives on Psychological Science, 9, 666-681.

Simonsohn, U., Nelson, L. D., \& Simmons, J. P. (2014b). p-Curve: A key to the file-drawer. Journal of Experimental Psychology: General, 143, 534-547.

Smith, M. L., \& Glass, G. V. (1977). Meta-analysis of psychotherapy outcome studies. American Psychologist, 32, 752.

Stanley, T. D. (2017). Limitations of PET-PEESE and other meta-analysis methods. Social Psychological and Personality Science, 8, 581-591.

Stanley, T. D., \& Doucouliagos, H. (2014). Meta-regression approximations to reduce publication selection bias. Research Synthesis Methods, 5, 60-78.

Stanley, T. D., Jarrell, J. B., \& Doucouliagos, H. (2010.) Could it be better to discard 90\% of the data? A statistical paradox. The American Statistician, 64, 70-77.

Stegenga, J. (2011). Is meta-analysis the platinum standard of evidence? Studies in history and philosophy of science part C: Studies in history and philosophy of biological and biomedical sciences, 42, 497-507.

Sterling, T. D. (1959). Publication decisions and their possible effects on inferences drawn from tests of significance - Or vice versa. Journal of the American Statistical Association, 54, $30-34$.

Sterne, J. A. C., Egger, M., Moher, D., \& Boutron, I. (2017, June). Chapter 10: Addressing reporting biases. In J. P. T. Higgins, R. Churchill, J. Chandler, M. S. Cumpston (eds.), 
Cochrane Handbook for Systematic Reviews of Interventions version 5.2.0. Retrieved from www.training.cochrane.org/handbook.

Ulrich, R., \& Miller, J. (2015). p-hacking by post hoc selection with multiple opportunities:

Detectability by skewness test? Comment on Simonsohn, Nelson, and Simmons (2014). Journal of Experimental Psychology: General, 144, 1137-1145.

van Aert, R. C. M., Wicherts, J. M., \& van Assen, M. A. L. M. (2016). Conducting metaanalyses based on $p$-values: Reservations and recommendations for applying $p$-uniform and p-curve. Perspectives on Psychological Science, 11, 713-729.

van Assen, M. A., van Aert, R., \& Wicherts, J. M. (2015). Meta-analysis using effect size distributions of only statistically significant studies. Psychological Methods, 20, 293309.

Vohs, K. D. (2018, March 3). A pre-registered depletion replication project: The paradigmatic replication approach. Presented at the Annual Convention of the Society of Personality and Social Psychology, Atlanta, GA.

Woll \& Schönbrodt, F. (In press). A series of meta-analytic tests of the efficacy of long-term psychoanalytic psychotherapy. European Psychologist. doi: https://doi.org/10.1027/1016$\underline{9040 / \mathrm{a} 000385}$ 